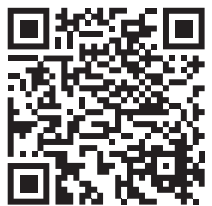

Palabras clave: Simulación, carga mental, habilidades no técnicas, COVID-19, rendimiento clínico, autopercepción de eficacia, traslacional.

Keywords: Simulation, cognitive load, non technical skills, COVID-19, clinical performance, perception of selfefficacy, translational.

* INSPIRE Simulación FEMEBA.

₹ Sociedad Platense de Anestesiología.

$\S$ Centro de Simulación

Clínica Avanzada de la Universidad Francisco de Vitoria de Madrid.

FEMEBA.

Recibido: 15/06/2020

Aceptado: 15/07/2020

doi: $10.35366 / 95233$

\title{
Atención de un paciente sospechoso de infección por COVID-19: análisis de un caso de simulación clínica avanzada
}

\author{
Care of a patient suspected of COVID-19 infection: \\ a high fidelity simulation case analysis
Juan Ignacio Cobián, ${ }^{*}$ Federico Ferrero, ${ }^{\ddagger}$ Mario Alberto Fontana, ${ }^{*}$ Magdalena Aprea,* Salvador Ramírez-Espinosa ${ }^{\S}$

\section{RESUMEN}

Introducción: La experiencia clínica simulada permite evaluar la respuesta de equipos de atención prehospitalaria, urgencias y cuidados críticos frente a un caso sospechoso de COVID-19. Presentación del caso: Se dispuso un entorno múltiple que comenzó en el domicilio y continuó en un hospital. Durante el análisis se realizó un ejercicio de autoevaluación sobre emociones, carga mental y autopercepción de eficacia. Se encontró una diferencia en la carga mental en favor de los grupos de cuidados críticos y urgencias en comparación con atención prehospitalaria. Los tres grupos expresaron elevada APE; sin embargo, se observó una brecha de rendimiento en "prevención y control de la infección" y "habilidades no técnicas". Conclusiones: Resaltamos la necesidad de tener en cuenta la carga mental y las habilidades no técnicas en el diseño de la experiencia clínica simulada, su rol en la autopercepción de eficacia, y el valor de la simulación traslacional en la mejora continua de procesos para la atención del COVID-19.

\section{ABSTRACT}

Introduction: Simulated clinical experience allows to asses response of pre-hospital care, emergencies and critical care teams to a suspected case of COVID-19. Case report: A multiple environment was set, beginning at patient's home and continued in hospital. During the analysis, a self-assessment exercise was carried out on emotions, cognitive load and self-perception of efficacy. A difference was found in the cognitive load in favor of the critical care and emergencies groups compared to pre-hospital care. All three groups expressed high self-perception of efficacy; however, a performance gap was observed in terms of prevention and control of the infection and nontechnical skills. Conclusions: We highlight the need to take cognitive load and non-technical skills into account in the design of simulated clinical experience, their role in self-perception of efficacy, and the value of translational simulation in the continuous improvement of processes for the care of COVID-19.

\section{INTRODUCCIÓN}

$E^{\prime}$ crecimiento geométrico de la morbimortalidad asociada al nuevo coronavirus (COVID-19) ha puesto a prueba a todos los sistemas de salud del mundo. En América Latina se ha propagado a los 54 países y territorios de la región.

Diferentes organismos han desarrollado programas de capacitación para el personal de salud.2,3 La mayoría de estos programas no han incluido al aprendizaje situado ${ }^{4}$ dentro de sus estrategias, perdiéndose la oportunidad de descubrir y mejorar aquellos aspectos no técnicos que tanto influyen en el rendimiento de los equipos. En países como Argentina, las tasas de contagio de los profesionales de la salud se mantienen elevadas, ubicándose alrededor del $12.5 \%$ de los casos confirmados. ${ }^{5}$

El objetivo de esta experiencia clínica simulada fue evaluar la respuesta de equipos de profesionales de la salud correspondientes a diferentes niveles de atención frente a un caso sospechoso de COVID-19, poniendo énfasis en los factores humanos involucrados.

\section{PRESENTACIÓN DEL CASO}

La experiencia clínica simulada que presentamos se realizó unos pocos días antes de la implementación del aislamiento social preventivo y obligatorio en la República Argentina. El diseño del caso, la dinámica de trabajo y la logística tomó un mes de 
trabajo previo, y fue basado en INACSL Standards of Best Practice: Simulation ${ }^{\text {SM }} 6$

Toda la experiencia fue difundida mediante streaming a través de Zoom.us (Zoom Video Communications, Inc.) y Facebook Live para facilitar el intercambio con expectadores de diferentes puntos de Iberoamérica. El objetivo general: "evaluar la atención de un paciente sospechoso de COVID-19". En virtud de este objetivo, se evaluaron tres grandes aspectos del rendimiento de los participantes. Para cada uno de ellos se dispuso una lista de verificación específica. Los mismos se especifican a continuación:

1. Clínicos: atención clínica adecuada.

2. Prevención y control de la infección: control de la exposición del personal y otros pacientes, utilización adecuada de los equipos de protección personal, toma y conservación de la muestra, higiene de manos y seguridad en los procedimientos invasivos.

3. E-CRM: ${ }^{7}$ comunicación, ayuda y gestión de la ayuda, uso de recursos y claridad de papeles.

Finalmente, se realizó una evaluación global: un total de 11 profesionales pertenecientes a tres equipos de trabajo, prehospitalaria, urgencias y cuidados críticos participaron de la experiencia: un técnico en emergencias, cinco enfermeros y cinco médicos. Diez de los 11 participaban por primera vez de una simulación.

El caso inicia con un llamado al Servicio de Emergencias por parte de la esposa de un hombre de 50 años que se encuentra en su domicilio, recientemente llegado de zona epidémica, con un cuadro de rinitis y fiebre de 24 horas de evolución. Al momento de la comunicación ha evolucionado negativamente con dificultad para respirar, tos productiva y "temblores".

Se utilizaron tres simuladores para el mismo paciente: un paciente simulado y dos maniquíes,

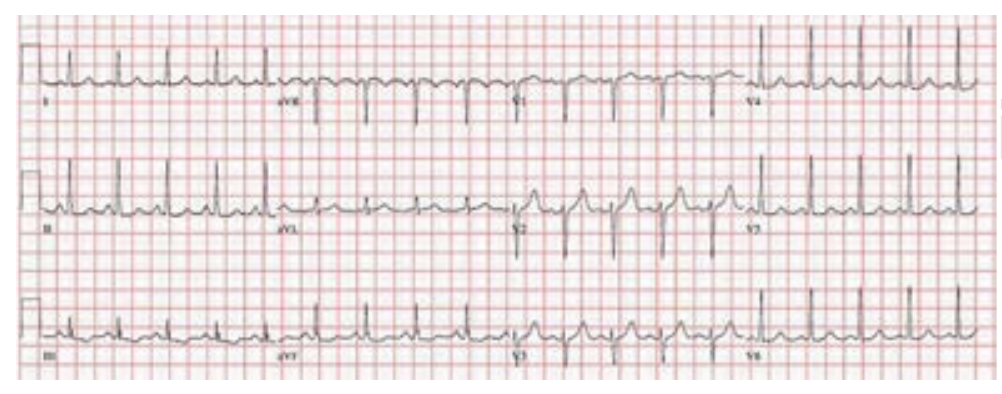

Figura 1: Electrocardiograma paciente sospechoso de COVID-19.

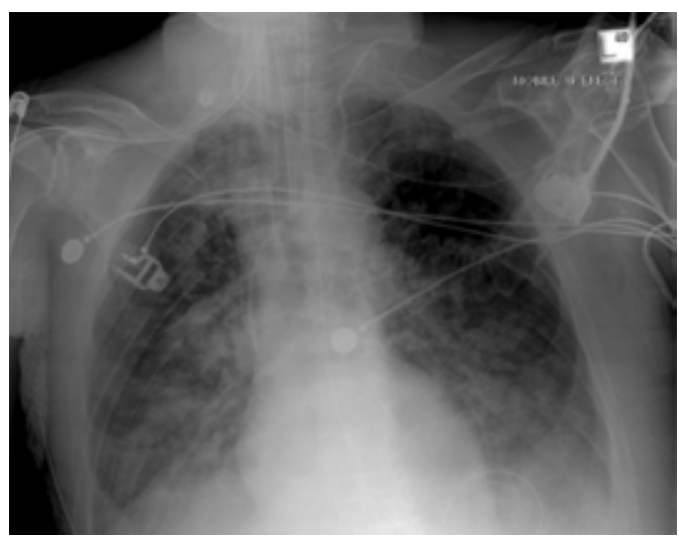

Figura 2: Rx tórax frente paciente sospechoso de COVID-19.

Apollo y i-Stan (CAE Healthcare, Inc.), según las etapas que fue cumpliendo la simulación, y un simulador virtual de ultrasonido Vimedix (CAE Healthcare Inc.) como método de estudio complementario.

Se dispuso un entorno múltiple para el desarrollo de la experiencia clínica simulada, con tres etapas, que comenzó en el domicilio y continuó en el ámbito de un hospital, desde el ingreso a la institución a través del servicio de urgencias hasta el área de cuidados intensivos. La presentación del ambiente de simulación se llevó a cabo en forma simultánea con cada uno de los equipos en el lugar correspondiente, y tuvo una hora de duración. Se hizo especial hincapié en el cambio de simulador para mitigar la carga cognitiva extraña. La finalización de las primeras dos etapas estaba prevista por objetivos o por tiempo tras la activación de prompts por parte de los confederados. El tiempo fue el único criterio de finalización de la última etapa. La duración de todo el escenario fue de 35 minutos. Los estudios complementarios se detallan en las Figuras 1 y 2.

Participaron un especialista en infectología y dos en emergencias sanitarias como observadores de la simulación y en forma activa durante el análisis posterior. El personal involucrado se detalla en la Tabla 1.

Para el análisis se optó por un debriefing tipo Pearls $(\mathrm{EPPICH})^{8}$ que tuvo una duración de 90 minutos. Durante el mismo, se otorgó a los participantes una planilla que contenía dos instrumentos de autovaloración. El primero de ellos constituye una tipología de emociones, de la cual se solicitó a los participantes que se reconocieran e identificarán en alguna de ellas. El segundo instrumento utilizado fue un cuestionario de seis preguntas tomadas de la escala subjetiva de carga 
mental de trabajo (ESCAM) $)^{9}$ y cuyas respuestas debían ubicarse en una escala de Likert de cinco opciones (muy bajo a muy alto). Se indagó sobre exigencia mental, temporal y física, rendimiento, esfuerzo y frustración (Figura 3). Por último, se

\begin{tabular}{lcl}
$\begin{array}{c}\text { Tabla 1: Se detallan los recursos humanos requeridos } \\
\text { para llevar a cabo la experiencia clínica simulada. }\end{array}$ \\
\hline Rol & Número & Función \\
\hline $\begin{array}{l}\text { Confederados } \\
\text { Facilitadores }\end{array}$ & 5 & $\begin{array}{l}\text { Esposa, enfermeras y técnico } \\
\text { Tres para la ejecución de cada escenario } \\
\text { Actores }\end{array}$ \\
$\begin{array}{l}\text { Paciente simulado } \\
\text { Técnicos } \\
\text { Operativos }\end{array}$ & 5 & $\begin{array}{l}\text { Seguridad, limpieza, pacientes y médico } \\
\text { Paciente en etapa prehospitalaria } \\
\text { Difusión online } \\
\end{array}$ \\
\end{tabular}

hizo un feedback directo sobre elementos relacionados con habilidades técnicas concretas, por ejemplo, manejo de la vía aérea en un paciente con sospecha de COVID.

\section{DISCUSIÓN}

Hemos llevado a cabo una experiencia clínica simulada de tipo híbrida, principalmente correspondiente a la zona 3 de la clasificación de Roussin. ${ }^{10} \mathrm{Si}$ bien el cuestionario autoadministrado no se encuentra validado, su análisis señala una diferencia en la carga de trabajo cognitivo (en seis de los cinco ítems), en favor de los grupos de participantes de cuidados críticos y urgencias en comparación con los participantes de atención prehospitalaria que realizaron la atención domiciliaria. Con respecto al rendimiento durante el escenario (percepción de autoeficacia), los tres grupos coincidieron en expresar una alta

\section{Tipografía de emociones.}

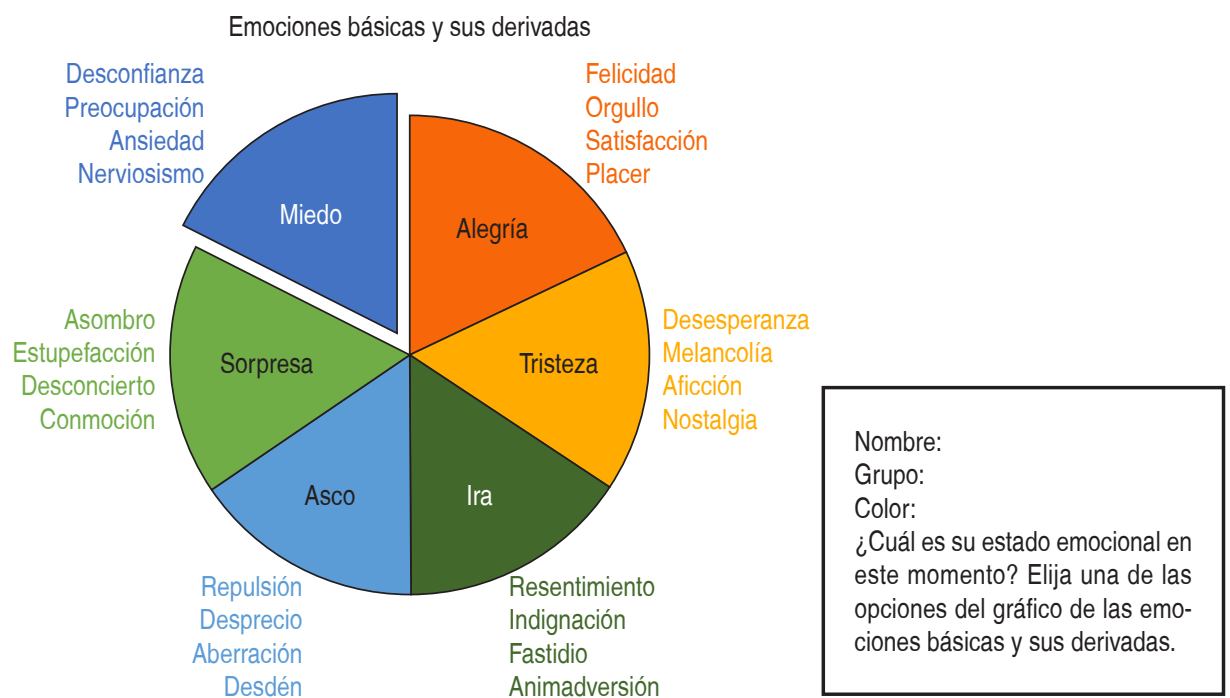

$$
\begin{aligned}
\text { Muy bajo } & =1 \\
\text { Bajo } & =2 \\
\text { Medio } & =3 \\
\text { Alto } & =4 \\
\text { Muy alto } & =5
\end{aligned}
$$

Figura 3:

Instrumentos de autoevaluación para la fase de análisis de la experiencia clínica simulada.

\section{Cuestionario adaptado de escala subjetiva de carga mental.}

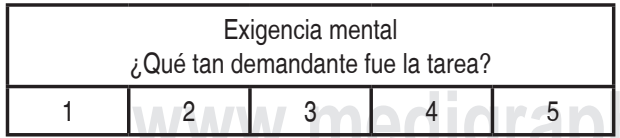

Exigencia temporal

\begin{tabular}{|c|c|c|c|c|}
\hline \multicolumn{5}{|c|}{ Exigencia temporal } \\
¿Qué tan rápido fue el ritmo impuesto para hacer la tarea? \\
\hline 1 & 2 & 3 & 4 & 5 \\
\hline
\end{tabular}

Esfuerzo

¿Qué tan duro tuvo que trabajar para lograr el punto 4?

\begin{tabular}{|l|l|l|l|l|}
\hline 1 & 2 & 3 & 4 & 5 \\
\hline
\end{tabular}

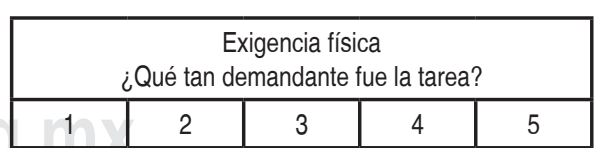

Rendimiento ¿Qué tan exitoso ha sido para lograr lo requerido?

\begin{tabular}{l|l|l|l|l}
1 & 2 & 3 & 4 & 5 \\
\hline
\end{tabular}

Frustración

¿Qué tan inseguro, irritado, estresado o molesto está?

\begin{tabular}{|l|l|l|l|l|}
\hline 1 & 2 & 3 & 4 & 5 \\
\hline
\end{tabular}


valoración sobre su actuación. Al comparar esta percepción con los instrumentos de registro utilizados por los observadores, hallamos una brecha de rendimiento en aspectos relacionados con los objetivos de prevención y control (manejo de elementos de protección personal, toma de muestras) y habilidades no técnicas.

\section{CONCLUSIONES}

Creemos que la experiencia realizada contribuye al desarrollo de la simulación de alta fidelidad en varios aspectos. En primer lugar, pone de manifiesto la necesidad de tener en cuenta la carga mental a la hora de diseñar una experiencia clínica simulada. ${ }^{11}$ Luego, evidencia que las habilidades no técnicas requeridas para el abordaje de situaciones complejas habitualmente no son consideradas, y esto podría influir en la forma en que nos percibimos como profesionales y en nuestros resultados. ${ }^{12}$ Por último, nos interesa recuperar el valor de este escenario en términos de su aporte a la mejora continua de procesos a través de la simulación traslacional. En particular, creemos que esta simulación puede ubicarse en la etapa diagnóstica de la misma, ${ }^{13}$ con potencialidad para mejorar la calidad de atención en el marco de la pandemia de COVID-19.

\section{AGRADECIMIENTOS}

Agradecemos a los equipos de profesionales de la salud que han tenido el valor de participar mostrándose tal y como son, y por su interés en mejorar en lo científico-técnico y humano.

\section{REFERENCIAS}

1. https://www.paho.org/es/tag/informes-situacion-paracovid-19.
2. https://www.paho.org/es/noticias/30-3-2020-opslanza-tres-cursos-sobre-covid-19-espanol.

3. https://openwho.org/courses/COVID-19-PCI-ES.

4. Mann KV. Theoretical perspectives in medical education: past experience and future possibilities. Med Educ. 2011; 45 (1): 60-68.

5. https://portal-coronavirus.gba.gob.ar/sala-de-situacion.

6. Casal AC, Quintillá MJ, Espinosa RS. Simulación clínica y seguridad en urgencias y emergencias. Emergency Crisis Resource Management (E-CRM). Emergencias. 2020; 32(2): 135-137.

7. INACSL Standards Committee (2016, December). INACSL Standards of Best Practice: Simulation ${ }^{\mathrm{SM}}$ : Simulation design. Clinical Simulation in Nursing. 2016; 12: S5-S50. https://doi.org/10.1016/j. ecns.2016.09.009.

8. Eppich W, Adam C. Promoting Excellence and Reflective Learning in Simulation (PEARLS). Simul Healthc. 2015; 10 (2): 106-115. doi: 10.1097/ SIH.0000000000000072.

9. Rolo GG, Díaz CD, Hernández FE. Desarrollo de una escala subjetiva de carga mental de trabajo (ESCAM). Rev Psicol Trab Organ. 2009; 25 (1): 29-37.

10. Roussin CJ, Weinstock P. SimZones: An organizational innovation for simulation programs and centers. Acad Med. 2017; 92 (8): 1114-1120. doi: 10.1097/ ACM.0000000000001746.

11. Leppink J, Van den Heuvel A. The evolution of cognitive load theory and its application to medical education. Perspect Med Educ. 2015; 4 (3): 119-127. doi: 10.1007/s40037-015-0192-x.

12. Penal G. Surgeons' and trainees' perceived self-efficacy in operating theatre non-technical skills. Br J Surg. 2015; 102: 708-715. doi: 10.1002/bjs.9787.

13. Brazil V. Translational simulation: not 'where?' but 'why?' A functional view of in situ simulation. Adv Simul. 2017; 20 (2): 20. https://doi.org/10.1186/ s41077-017-0052-3.

Correspondencia: Juan Ignacio Cobián

Calle 54 Núm. 920, La Plata, Argentina. E-mail: jcobian@inspirefemeba.com.ar juanignaciocobian@gmail.com 